\title{
Direct Spherical Azimuth Angles Determination
}

\author{
Sebahattin Bektas \\ 19Mayls University, Faculty of Engineering, Geomatics Department, Samsun
}

*Corresponding Author: Sebahattin Bektas, 19Mayls University, Faculty of Engineering, Geomatics Department, Samsun

\begin{abstract}
In this paper, we will see that the determination of direct bearing angles. As it is known, in bearing angles are often computed used formulas with arctan function. The arctan function gives an angle values between $-90^{\circ}$ and $+90^{\circ}$. However, the bearing angle is by definition $0^{\circ}$ to $360^{\circ}$. Consequently, it is inevitable to examine the process of obtaining the azimuth angle.

Classic formulas only work correctly if the edge is in the 1st quarter. If the edge is located in the other quarters, the angles of the bearing should be examined. In this work we proposed a new formulas for direct bearing angles on globe (sphere). Using the formula that we propose will save execution time in codes with intensive geodesic calculations.
\end{abstract}

Keywords: Azimuth Angles, Sphere, Geographical coordinates

\section{INTRODUCTION}

For example, First Geodetic Basic problem; $\mathrm{P}_{1}\left(\varphi_{1}, \lambda_{1}\right)$ the geographic coordinates of a point $\mathrm{P} 1$ are given in latitude longitude values, $\mathrm{S}_{12}$ the geodetic curve length from point $\mathrm{P} 1$ to point $\mathrm{P} 2, \mathrm{~A}_{12}$ theazimuth angle (bearingangle) of the length and desiredP $\mathrm{P}_{2}\left(\varphi_{2}, \lambda_{2}\right)$ the geographic coordinates of a point $\mathrm{P} 2$. The azimuth $\mathrm{A}_{21}$ is desirable which is corresponding $\mathrm{A}_{12}$ azimuth angle. Because there is approximately 180 degrees difference between $A_{12}$ and $A_{21}$. Thus, the region of $A_{21}$ is easily predicted. If the two points are on the same meridian oron the same parallel circle the difference between $\mathrm{A}_{12}$ and $\mathrm{A}_{21}$ is exactly $180^{\circ}$ degrees [5],[6].

However, in the 2nd Geodetic basic problem; the geographic coordinates latitude and longitude values of the two points are given; $\mathrm{P}_{1}\left(\varphi_{1}, \lambda_{1}\right), \mathrm{P} 2\left(\varphi_{2}, \lambda_{2}\right)$ and required the geodesic curve length between the two points is $S_{12}$ and the corresponding azimuths $A_{12}$ and $A_{21}$ between the two points. The azimuth calculation is not as easy as in the $1^{\text {st }}$ Geodetic basic problem assignment. If the $A_{12}$ azimuth is calculated incorrectly, the $\mathrm{A}_{21}$ azimuth will also be incorrect by itself.

In this proposed method, formulas are given for how to obtain the azimuth angle directly without any examination. The given method can calculate azimuth without reducing the sphere and ellipsoid surface.

\section{MAterial AND Methods}

Calculation of between the two points $S_{12}$ and the corresponding azimuths $A_{12}$ and $A_{21}$ from known $\mathrm{P} 1, \mathrm{P} 2$ points geographical coordinates is also called as geodetic $2^{\text {nd }}$ basic problem solution (see Fig.1). Problem is solved classically with below formulas[1], [2], [4] 


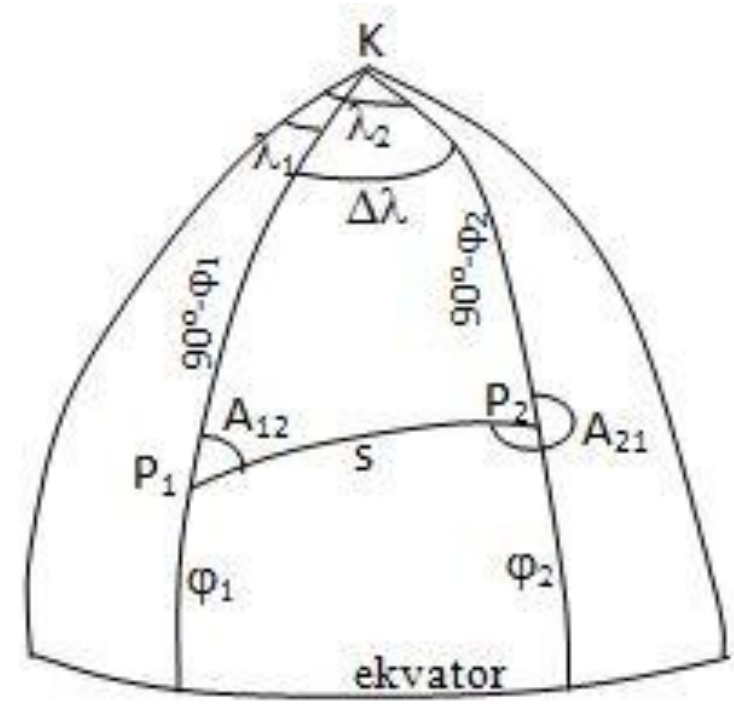

Figure1.

Classic Method;

$\sigma=\arccos \left(\sin \varphi_{1} \sin \varphi_{2}+\cos \varphi_{1} \cos \varphi_{2} \cos \Delta \lambda\right.$

$\mathrm{A}_{12}=\arctan \left(\frac{\sin \Delta \lambda}{\tan \varphi_{2} \cos \varphi_{1}-\sin \varphi_{1} \cos \Delta \lambda}\right)$

$\mathrm{A}_{21}=\arctan \left(\frac{\sin \Delta \lambda}{\cos \Delta \lambda \sin \varphi_{2}-\cos \varphi_{2} \tan \varphi_{1}}\right)+\pi$

Here $\sigma$ is the angular equivalent of the edge. If you want to find the metric of the edge

$\mathrm{S}=\sigma / \rho . \mathrm{R} \quad \rho=180^{\circ} / \Pi \quad \mathrm{R}:$ radius of the earth

It is important to remember that these classic formulas only work correctly if the edge is in the 1st quarter. If the edge is located in the other quarters, the angles of the bearing angle should be examined. For correct angles, the necessary additions should be made according to the Table-1 below.

Table1. Fixed value to add for bearing angles

\begin{tabular}{|c|c|c|}
\hline Quadrant & $\begin{array}{c}\text { fixed value to add } \\
\text { for } \mathrm{A}_{12}\end{array}$ & $\begin{array}{c}\text { fixed value to add } \\
\text { for } \mathrm{A}_{21}\end{array}$ \\
\hline 1.Quadrant & - & - \\
\hline 2.Quadrant & $+180^{\circ}$ & $+180^{\circ}$ \\
\hline 3.Quadrant & $+180^{\circ}$ & $-180^{\circ}$ \\
\hline 4.Quadrant & $+360^{\circ}$ & - \\
\hline
\end{tabular}

Proposed Method

For direct determination of azimuth by geographic coordinates, we give below formulas. In this proposed method, formulas are given how to obtain the azimuth angle directly without any examination. The proposed method can calculate direct azimuth angles on the sphere and ellipsoid surface [3].

$$
\begin{gathered}
\mathrm{I}=\sin \Delta \lambda \\
\mathrm{II}=\tan \phi_{2} \cos \phi_{1}-\sin \phi_{1} \cos \Delta \lambda \\
\mathrm{A}_{12}=2 \cdot \arctan \left(\frac{I}{I I-\sqrt{I^{2}+I I^{2}}}\right)+180^{\circ} \\
\mathrm{III}=\tan \phi_{1} \cos \phi_{2}-\sin \phi_{2} \cos \Delta \lambda \\
\mathrm{A}_{21}=2 \cdot \arctan \left(\frac{I}{-I I I+\sqrt{I^{2}+I I I^{2}}}\right)+180^{\circ}
\end{gathered}
$$




\section{Numerical Example}

To compare direct formula and classical formula results, From the point P1 to the point P2 which is located in different quarters each time, the second basic problem solutions were made and the bearing angles calculations were made.

$$
\begin{aligned}
& \mathrm{P}_{1}\left(\varphi_{1}, \lambda_{1}\right) \text { the geographic coordinates of a point } \mathrm{P} 1 \text { are given in latitude longitude values: } \\
& \varphi_{1}=30^{\circ}, \lambda_{1}=30^{\circ} \\
& \text { Required: } \quad \mathrm{S}, \mathrm{A}_{12}, \mathrm{~A}_{21}
\end{aligned}
$$

If we use the above classic method formula and proposed method formula for the solution, for results please see Table-2

Table2. Direct formula and classical formula results

\begin{tabular}{|c|l|l|l|l|l|l|l|}
\hline \multicolumn{9}{|c|}{} & \multicolumn{2}{l|}{ Classic Formula } & \multicolumn{2}{c|}{ Proposed (Direct) Formula } \\
\hline Quadrant & $\varphi_{2}$ & $\lambda_{2}$ & \multicolumn{1}{c|}{$\mathrm{S}$} & \multicolumn{1}{|c|}{$\mathrm{A}_{12}$} & $\mathrm{~A}_{21}$ & $\mathrm{~A}_{12}$ & $\mathrm{~A}_{21}$ \\
\hline 1 & 32 & 31 & 241911.948 & 22.94320 & 203.45833 & 22.94320 & 203.45833 \\
\hline 2 & 29 & 32 & 223183.087 & -60.61890 & 120.36606 & 119.38110 & 300.36606 \\
\hline 3 & 28 & 29 & 242683.026 & 23.86428 & 203.37939 & 203.86428 & 23.37939 \\
\hline 4 & 32 & 29 & 241911.948 & -22.94320 & 156.54167 & 337.05680 & 156.54167 \\
\hline
\end{tabular}

\section{RESUlT AND DiscUSSION}

In this proposed method, formulas are given for how to obtain the azimuth angle directly without any examination. The given method can calculate azimuth without reducing the sphere and ellipsoid surface.

The numerical example that we have given shows the accuracy of the method we propose. The advantage of the method is that no examination is required. In computer calculations, if...end blocks are not used when direct formulas are used. The if...end blocks reduce the execution speed in computer calculations. For future studies, researchers are advised to try to find more simple direct formulas.

\section{CONCLUSION}

In this proposed method, formulas are given for how to obtain the azimuth angle directly without any examination. The given method can calculate azimuth without reducing the sphere and ellipsoid surface. Using the formula that we propose will save execution time in codes with intensive geodesic calculations

\section{REFERENCES}

[1] Bektaş, S. (2001) :" MatematikJeodezi ", Kamer MatbaaYayıncılık,Samsun

[2] Bektaş, S. (2004) : "Jeodezik Hesap-DüzlemdeveKüreYüzeyinde Jeodezik Hesaplamalar", OMÜ yayınlar1, Yayın no:120,Samsun

[3] Bektaş, S. (2016) :" Pratik Jeodezi",2.Baskı, OMÜ yayınları, Yayın no:120,Samsun

[4] Yaşayan,A.,Hekimoğlu,Ş. (1982): "KüreselTrigonometri”, KTÜ yayınları, Yayın no : 143/122, Trabzon

[5] Grossmann, W. (1964) :"GeodaticheRechnungen und Abbildungen in der Landesvermessung”, Verlag Konrad Wittwer, Stutgart 1964

[6] Hristow,W.K.(1943):’Die Gauss-Krüger'schenKoordinaten auf dem Ellipsoid”,Berlin

Citation: Sebahattin Bektas, (2020)"Direct Spherical Azimuth Angles Determination", International Journal of Mining Science (IJMS), 6(3), pp. 1-3. DOI: http://doi.org/10.20431/2454-9460.060 3001

Copyright: (C) 2020 Sebahattin Bektas, This is an open-access article distributed under the terms of the Creative Commons Attribution License, which permits unrestricted use, distribution, and reproduction in any medium, provided the original author and source are credited. 